\title{
Tourists Can Enjoy a Rainbow Every Sunny Day
}

\author{
Florin D.Grosu' ${ }^{1}$, Alina Rapa ${ }^{2}$, Ilie Diaconu ${ }^{3}$, Ioan Grosu ${ }^{3,4, "}$ \\ ${ }^{1}$ Faculty of Medicine,University Lucian Blaga, Sibiu, Romania \\ ${ }^{2}$ ACP Department,KwantlenPolytechnicUniversity, Metro Vancouver, Canada \\ ${ }^{3}$ Medical Bioengineering, University of Medicine andPharmacyGr.T.Popa, Iasi \\ ${ }^{4}$ Chemistry Department, Al.I.Cuza University, Iasi, Romania
}

*Corresponding Author: Ioan Grosu, Medical Bioengineering, University of Medicine and Pharmacy Gr.T.Popa, Iasi, Chemistry Department, Al.I.Cuza University, Iasi, Romania

\begin{abstract}
Anytime and any where a rainbow brings wonder, better mood, enthusiasm and well-being and many smiles. We present a simple and cheap installation that allows to obtain an artificial rainbow that is very similar with the natural one.On any sunnyday, tourists can enjoy a rainbow. This can increase the attractiveness of touristic places by innovation and the tourists satisfaction.
\end{abstract}

Keywords: tourism, innovation in tourism, touristic places, attractiveness of touristic places, rainbow, tourists satisfaction.

\section{INTRODUCTION}

Rainbow is a natural phenomenon related to Optics (Physics) and Metheorology. It is mentioned even in Bible in connection with Flood and Noah s Ark/1/. People enjoy looking at a rainbow and there are some superstitions about it. It is said that rainbow brings luck but for sure it brings wonder, better mood, enthusiasm, well-being and many smiles. People like to take pictures like for a special happening. The first full scientific explanation of how a rainbow is formed was given by the french scientist Rene Descartes (1596-1650)/2/. This theory is at the level of upper classes of high school at Physics/3/.Advanced research results are available in the scientific literature /4,5/.

If we are lucky enough we can see a rainbow one or two times per year. Descartes theory gives a practical result: a rainbow can be seen if an observer looks to a curtain of rain drops and behind $\mathrm{him} / \mathrm{her}$ the Sun is at 42 degrees on a clear sky. It means that half of the sky should be covered by clouds (that produce a rain) and the other half of the sky should be clear and the Sun should be at 42 degrees above the horizon. We can accept that these conditions are not so easy to be fulfilled and this is why we can see a rainbow one or two times per year. The Sun is at 42 degrees in the sky before noon (at approximate 10:30 AM) and afternnon (at 4:30 PM). The hours are not the same during the year.

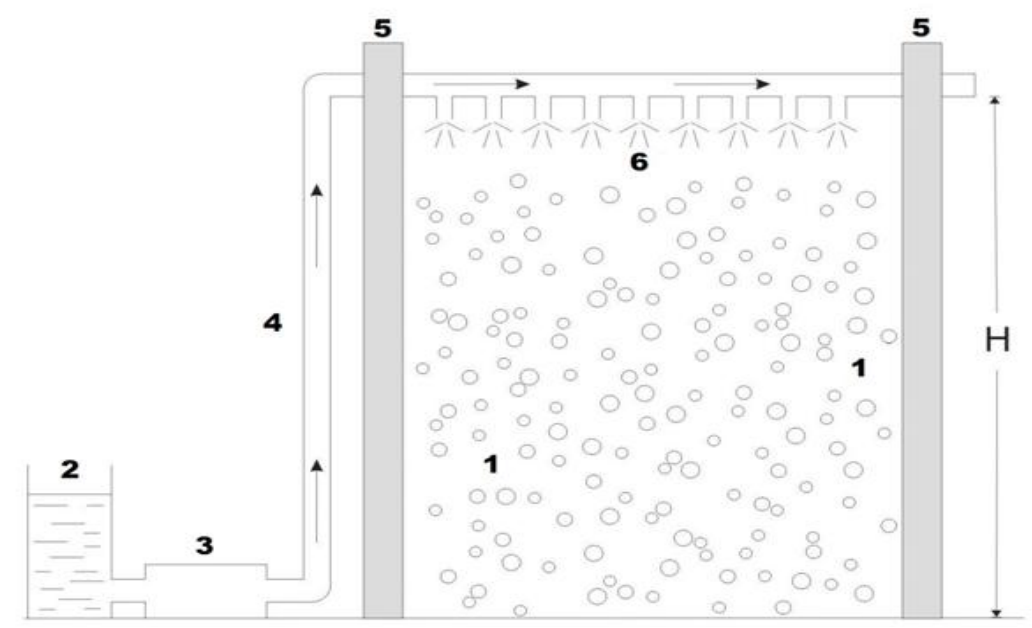

Figure1: Schematic of the installation. (1)-Water drops, (2)-vase with water, (3)-pump, (4)-tube, (5)-pillars, (6)sprayers 
In this short report we will present an installation that allows to obtain an artificial rainbow very similar with the natural one. Actually we produce artificially a rain (more exactly a curtain of water drops). This means that a rainbow can be seen every sunny day. The installation is presented schematically in Figure 1.

A tube is suspended, by some pillars, at a height $\mathrm{H}$ greater than $4 \mathrm{~m}$, its length is more than two times $\mathrm{H}$. On it are mounted some sprayers that produce water drops. There are several types of sprayers on the market. What is important is that water drops should have diameter less than $2 \mathrm{~mm}$ (otherwise the theory is not valid anymore). The water in tube is pumped by a pump or comes from the water net. Around a fountain we can see a small piece of a rainbow in a summer day. A gardener has much experience with the pieces of rainbow when he/she water his/hers plants. In this case water drops do not make a homogenous layer but one very in homogenous. In our case the curtain of water drops is homogenous and the colours are of the same intensity along the rainbow.

With such an installation we obtained a rainbow from Picture 1.

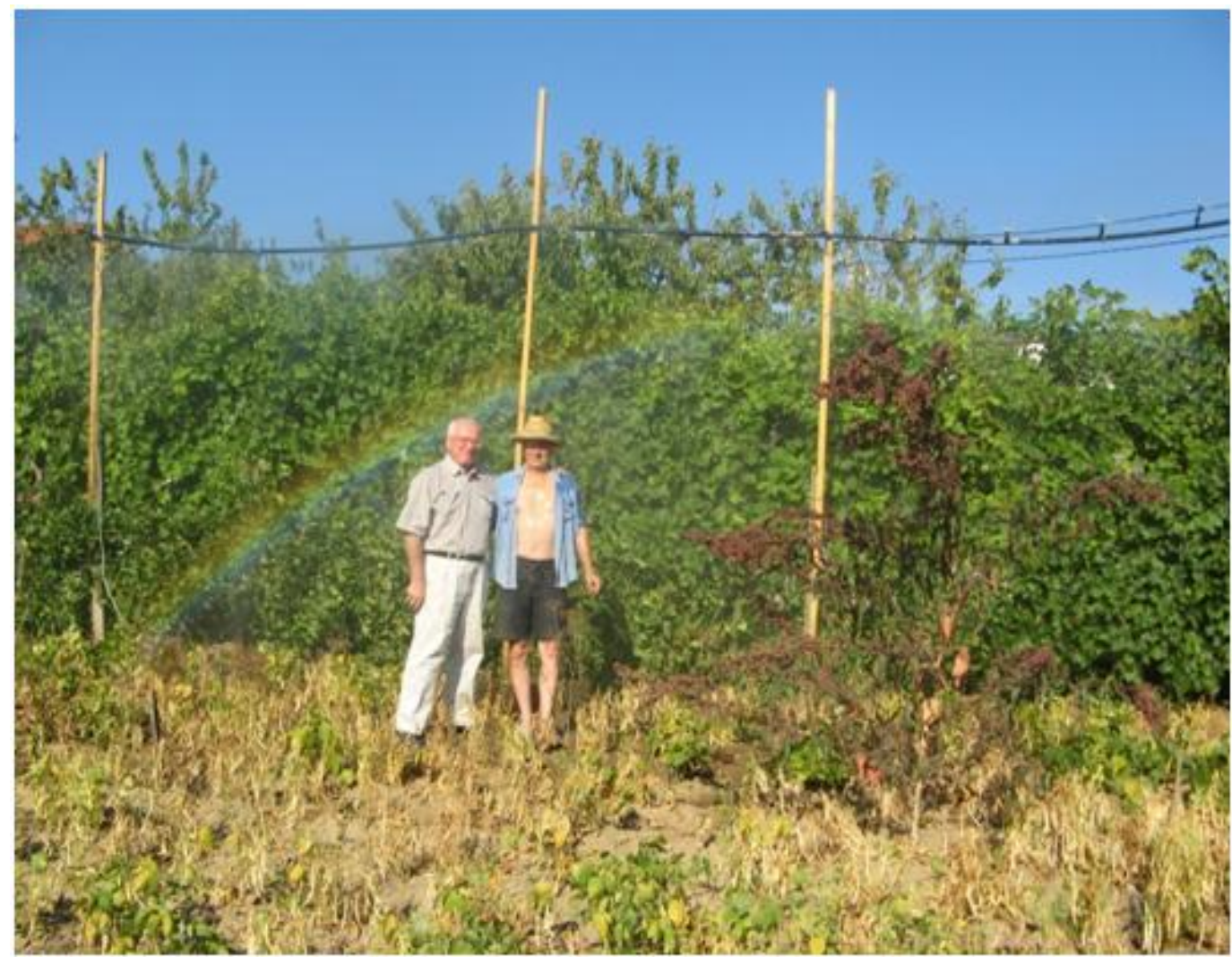

Picture1: The authors I.G. (left) and I.D. (right) in front of the rainbow in the garden of I.D.

Looking at the picture the first complaint is that the colors are too weak. To obtain more intense colors we need to produce more water drops. This can be realized by adding one or two or three tubes. Our installation costs less than 50 Euro. With 200 Euro a much nicer rainbow can be realized. The dimensions are not critical. What is important is that a tube with sprayers to be above (as much as possible) the soil. This can be realized with pillars (as in our Picture), between 2 houses or two trees or two hills or two mountains (above a narrow valley) etc. A good idea is to build it on a beach above the water (no inconveniences of a mud from water drops).

Tourists are happy to have exotic pictures and a selfie under a rainbow is the best for a memorable holiday. The authors are happy to answer any questions from the readers (ioan.grosu@chem.uaic.ro).

\section{REFERENCES}

[1] http: //en.wikipedia.org/wiki/Rainbow

[2] https://en.wikipedia.org/wiki/Rene_Descartes

[3] Florin D.Grosu, Alina Rapa, Ilie Diaconu and Ioan Grosu ,"Garden Rainbow", Merit Research Journal of Education and Review(ISSN 2350-2282), Vol 5(10),pp 106-110, Oct. 2017 , available on line http: //meritresearchjournals.org/er/index.htm 
[4] Michael Vollmer and Robert Tammer ,"Laboratory experiments in atmospheric optics" Appl.Optics $37(9), 1557$ (1998)

[5] Alexander Haussmann. , "Rainbows in nature: recent advances in observation and theory".Eur. J. of Phys. 37063001 (2016)

Citation: Ioan Grosu, Florin D. Grosu, et.al. "Tourists Can Enjoy a Rainbow Every Sunny Day". International Journal of Research in Tourism and Hospitality (IJRTH), vol 5, no. 4, 2019, pp. 07-09. doi: http://dx.doi.org/10.20431/2455-0043.0504002.

Copyright: (1) 2019 Authors. This is an open-access article distributed under the terms of the Creative Commons Attribution License, which permits unrestricted use, distribution, and reproduction in any medium, provided the original author and source are credited. 